\title{
First report of tobacco curly shoot virus infecting Malvastrum coromandelianum in China
}

\author{
Pengbai $\mathrm{Li}^{1} \cdot$ Chenchen Jing ${ }^{1} \cdot \mathrm{Ke} \mathrm{Li}^{1} \cdot$ Jiang $\mathrm{Du}^{1} \cdot \mathrm{Gentu} \mathrm{Wu}^{1} \cdot$ Mingjun $\mathrm{Li}^{1} \cdot$ Xianchao Sun ${ }^{1} \cdot \mathrm{Ling} \mathrm{Qing}^{1}$ \\ Published online: 16 April 2018 \\ (C) Società Italiana di Patologia Vegetale (S.I.Pa.V.) 2018
}

Tobacco curly shoot virus (TbCSV), a monopartite begomovirus in the family Geminiviridae, mainly infects tobacco and tomato and some weeds. To determine whether TbCSV infects Malvastrum coromandelianum, 26 samples of $M$. coromandelianum with typical foliar yellow vein symptom were collected from Sichuan and Guangxi provinces of China in 2016. Total DNA was extracted and subjected to PCR detection using degenerate primer PA/PB for geminiviruses (Deng et al. 2010). A 500 bp DNA fragment was amplified from all the samples. Then a fragment of ca. $1.0 \mathrm{~kb}$ was obtained from two (SC776 and GX121) of the 26 samples using TbCSV specific primers $\mathrm{Y} 35 \mathrm{~F} 1 / \mathrm{Y} 35+10 \mathrm{R}$ (Qing and Zhou 2009). Sequence alignment showed the highest identity with isolate TbCSV-[China: Sichuan118:2009], 99.5 and 99.7\%, respectively. Based on the obtained sequences, a pair of primer TbCSV-full- $\mathrm{F}\left(5^{\prime}-\right.$ TGTGACTGGTGGACAATATG-3')/TbCSV-full-R(5'GTTGCATGCCATTTCCGAAG-3') were designed for amplification of full length TbCSV DNA. The complete DNA sequences of isolates SC776 and GX121 were determined to be composed of 2747 and 2746 nucleotides (nts), respectively

Ling Qing

qling@swu.edu.cn

1 Chongqing Key Laboratory of Plant Disease Biology, College of Plant Protection, Southwest University, Chongqing 400716, People's Republic of China
(GenBank accession Nos MF977704 and MF977705) and shared the highest sequence identities $(99.5$ and $99.7 \%$, respectively) with TbCSV-[China: Sichuan118:09]. With the universal abutting primer (Briddon et al. 2002) for betasatellite DNA, an amplicon of nearly 1300 bp was amplified from two samples. Sequence alignment showed that both betasatellites are $1354 \mathrm{nts}$ in size (MF977706 and MF977707), and shared the highest identity (99.6 and 99.7\%, respectively) with TbCSB-[China:Yunnan 35:01]. To our knowledge, this is the first report of TbCSV associated with betasatellite infecting Malvastrum coromandelianum.

Funding This research work was supported by National Natural Science Founding of China (Grant No. 31772127) and the Fundamental Research Funds for the Central Universities (Grant No. XDJK2017A006).

\section{References}

Briddon RW, Bull SE, Mansoor S, Amin I, Markham PG (2002) Universal primers for the pcr-mediated amplification of dna beta: a molecule associated with some monopartite begomoviruses. Mol Biotechnol 20:315

Deng D, Mcgrath PF, Robinson DJ, Harrison BD (2010) Detection and differentiation of whitefly-transmitted geminiviruses in plants and vector insects by the polymerase chain reaction with degenerate primers. Ann Appl Biol 125:327-336

Qing L, Zhou X (2009) Trans-replication of, and competition between, DNA beta satellites in plants inoculated with tomato yellow leaf curl China virus and tobacco curly shoot virus. Phytopathology 99:716-720 